\title{
The inverse spectral problem for the discrete cubic string
}

\author{
Jennifer Kohlenberg $\dagger$, Hans Lundmark $\ddagger$ and Jacek \\ Szmigielski \\ $\dagger$ Institute of Biomaterials and Biomedical Engineering, University of Toronto, \\ Toronto, ON, M5S 3G9, Canada \\ ‡Department of Mathematics, Linköping University, SE-581 83 Linköping, \\ Sweden \\ $\S$ Department of Mathematics and Statistics, University of Saskatchewan, 106 \\ Wiggins Road, Saskatoon, Saskatchewan, S7N 5E6, Canada \\ E-mail: jennifer.kohlenberg@utoronto.ca, halun@mai.liu.se, \\ szmigiel@math.usask.ca
}

\begin{abstract}
Given a measure $m$ on the real line or a finite interval, the cubic string is the third order ODE $-\phi^{\prime \prime \prime}=z m \phi$ where $z$ is a spectral parameter. If equipped with Dirichlet-like boundary conditions this is a nonselfadjoint boundary value problem which has recently been shown to have a connection to the Degasperis-Procesi nonlinear water wave equation. In this paper we study the spectral and inverse spectral problem for the case of Neumann-like boundary conditions which appear in a high-frequency limit of the Degasperis-Procesi equation. We solve the spectral and inverse spectral problem for the case of $m$ being a finite positive discrete measure. In particular, explicit determinantal formulas for the measure $m$ are given. These formulas generalize Stieltjes' formulas used by Krein in his study of the corresponding second order ODE $-\phi^{\prime \prime}=z m \phi$.
\end{abstract}

AMS classification scheme numbers: 34A55, 34B05, 34B20, 41A28

Submitted to: Inverse Problems 


\section{Introduction}

In this paper we study the third order nonselfadjoint spectral problem

$$
\begin{aligned}
& -\phi^{\prime \prime \prime}(x)=z m(x) \phi(x) \quad \text { for } x \in \mathbf{R}, \\
& \phi_{x}(-\infty)=\phi_{x x}(-\infty)=0, \quad \phi_{x x}(\infty)=0,
\end{aligned}
$$

which we refer to as the cubic string with Neumann-like boundary conditions. In particular we consider the case when $m(x)$ is a discrete measure (a finite linear combination of Dirac deltas), and the problem of reconstructing $m(x)$ given appropriate spectral data. The purpose of this introduction is to explain where this problem comes from and why it might be of interest.

Our starting point is the Degasperis-Procesi (DP) equation, which is the completely integrable nonlinear PDE

$$
u_{t}-u_{t x x}+4 u u_{x}=3 u_{x} u_{x x}+u u_{x x x} .
$$

It was discovered purely mathematically in a search for integrable equations [1, 2], but has been shown by Johnson [3 to play a role in shallow water wave theory similar to that of the Camassa-Holm $(\mathrm{CH})$ equation [4]

$$
u_{t}-u_{t x x}+3 u u_{x}=2 u_{x} u_{x x}+u u_{x x x} .
$$

By taking appropriate scaling limits one can derive new nonlinear equations which inherit some of the integrable structure of their ancestors. Consider in particular the high-frequency limit obtained by changing variables $x \mapsto \varepsilon x, t \mapsto \varepsilon t$, and then letting $\varepsilon \rightarrow 0$. For the $\mathrm{CH}$ equation (1.3) this gives the Hunter-Saxton equation [5, 6] for nematic liquid crystals,

$$
\left(u_{t}+u u_{x}\right)_{x x}=u_{x} u_{x x} .
$$

The DP equation (1.2), on the other hand, reduces in the high-frequency limit to

$$
\left(u_{t}+u u_{x}\right)_{x x}=0 \text {, }
$$

which we will refer to as the derivative Burgers equation. The name of course refers to the well known inviscid Burgers equation (or Riemann shock wave equation) $u_{t}+u u_{x}=0$, the prototype equation for studying shock waves. That (1.5), with the two extra derivatives, has a Lax pair is a fairly recent discovery; as far as we are aware, this was first shown by Qiao and Li [7] (who studied the whole integrable hierarchy that (1.5) belongs to).

A unified way of writing these four equations is

$$
m_{t}+m_{x} u+b m u_{x}=0,
$$

with $m$ and $b$ as in the following table:

\begin{tabular}{c|c|c} 
& $m=u-u_{x x}$ & $m=u_{x x}$ \\
\hline$b=2$ & Camassa-Holm & Hunter-Saxton \\
\hline$b=3$ & Degasperis-Procesi & derivative Burgers
\end{tabular}

(According to Dullin, Gottwald and Holm [8], the whole family (1.6) with $m=u-u_{x x}$ and $b \neq-1$ can be derived as an asymptotic limit of the shallow water equations. The $\mathrm{CH}$ and DP equations are the only integrable cases in this family. Moreover, (1.6) with $m=u-u_{x x}$ is included in the more general family $u_{t}+\left[\delta u+3 \gamma u^{2} / 2+u^{1-\omega}\left(u^{\omega} u_{x}\right)_{x}\right]_{x}+$ $\nu u_{t x x}=0$ studied by Rosenau [9]; take $1+2 \omega=b,-3 \gamma=b+1, \delta=0, \nu=-1$, and let $x \mapsto-x$. Another generalization of (1.6) is the pulson equation introduced by Fringer and Holm [10, 11.) 
A similar table summarizes the particular kinds of soliton-like solutions characteristic of these equations, as well as the spectral problems (including the type of boundary conditions) employed when computing these solutions using inverse spectral methods:

\begin{tabular}{c|c|c} 
& PEAKON SOLUTIONS & PIECEWISE LINEAR SOLUTIONS \\
\hline Discrete string & Camassa-Holm & Hunter-Saxton \\
\hline Discrete cubic string & Degasperis-Procesi & derivative Burgers \\
\hline & Dirichlet(-like) & Neumann(-like)
\end{tabular}

This requires perhaps some explanation. Peakons (peaked solitons) are solutions taking the form

$$
u(x, t)=\sum_{k=1}^{n} m_{k}(t) \exp \left(-\left|x-x_{k}(t)\right|\right)
$$

while the piecewise linear solutions are given by

$$
u(x, t)=\sum_{k=1}^{n} m_{k}(t)\left|x-x_{k}(t)\right|,
$$

where in each case the functions $x_{k}(t)$ and $m_{k}(t)$ (positions and momenta of the solitons) are required to satisfy a certain system of $2 n$ ODEs in order for $u(x, t)$ to satisfy the PDE. (See Theorem 2.3 below for the case of the derivative Burgers equation.)

It was discovered in 12 that the spatial equation in the Lax pair of the $\mathrm{CH}$ equation can be transformed by a change of variables to the string equation with Dirichlet boundary conditions,

$$
\begin{aligned}
& -\phi^{\prime \prime}(x)=z m(x) \phi(x) \quad \text { for }-1<x<1, \\
& \phi(-1)=\phi(1)=0,
\end{aligned}
$$

where $z$ is the spectral parameter (squared frequency), $m$ is the mass density and $\phi$ represents the amplitude of the harmonic mode corresponding to to that frequency. (This originates of course in separation of variables in the linear wave equation $v_{x x}=m v_{t t}$ describing small vibrations of a string with given mass density $m(x)$.) The peakon solutions are related to the case when the mass distribution is a sum of point masses (Dirac deltas); this is what is meant by the discrete string. Krein [13, 14, 15] studied the inverse spectral problem of the string equation (1.9) and showed in particular that in the discrete case the mass distribution can be recovered from spectral data using continued fractions of Stieltjes type [16. This was exploited in [17, 18 to solve the $\mathrm{CH}$ peakon ODEs explicitly and analyze the solutions in detail. Similarly, the inverse problem for the string equation on $\mathbf{R}$ with Neumann boundary conditions $\phi_{x}(-\infty)=\phi_{x}(\infty)=0$ was used in [19] to solve the ODEs for the piecewise linear solutions of the Hunter-Saxton equation. The inverse spectral theory for the simple looking ODE (1.9) is surprisingly rich in mathematical content. The most comprehensive account of the inverse problem for this equation, and also of Krein's work on the subject, is the monograph [20] by Dym and McKean.

Despite the superficial similarity, the DP equation has a mathematical structure quite different from that of the $\mathrm{CH}$ equation. In particular, the spatial equation in the Lax pair of the DP eqation is of third order instead of second. In 21] we showed how to transform it into the cubic string with Dirichlet-like boundary conditions,

$$
\begin{aligned}
& -\phi^{\prime \prime \prime}(x)=z m(x) \phi(x) \quad \text { for }-1<x<1, \\
& \phi(-1)=\phi_{x}(-1)=0, \quad \phi(1)=0 .
\end{aligned}
$$


This problem was studied in great detail in our previous paper [22, where the inverse problem was solved in the discrete case, leading to explicit solution formulas for the DP peakons.

In this paper we first briefly show how the derivative Burgers equation is related to the cubic string with Neumann-like boundary conditions (1.1). Then we will give a fairly complete account of the direct and inverse spectral theory of (1.1) in the case when $m(x)$ is a positive discrete measure. Some of the ideas used in [22] are developed further, and we also provide new shorter proofs for some key steps which perhaps appeared mysterious there. For the most part this paper is self-contained, with the exception of using some Heine-like multiple integral formulas proved in 22$]$ (see Appendix B) and postponing the proof of Theorem 4.20 to another paper.

\section{The derivative Burgers equation}

Here we give a short account of the basic facts about the derivative Burgers equation (1.5). First we show how it inherits the integrable structure of the DP equation (1.2). The following Lax pair for the DP equation was given in [2]:

$$
\left(\partial_{x}-\partial_{x}^{3}\right) \phi=z m \phi, \quad \phi_{t}=\left[z^{-1}\left(c-\partial_{x}^{2}\right)+u_{x}-u \partial_{x}\right] \phi,
$$

where $c$ is an arbitrary constant (or function of $z$ ); indeed, the compatibility condition $\phi_{t x x x}=\phi_{x x x t}$ is equivalent to $m_{t}+m_{x} u+3 m u_{x}=0$ and $m_{x}=\left(u-u_{x x}\right)_{x}$. The high-frequency substitution $x \mapsto \varepsilon x, t \mapsto \varepsilon t$, together with $m \mapsto-\varepsilon^{-2} m, z \mapsto-\varepsilon^{-1} z$, and $c \mapsto-z \varepsilon^{-2} c$, yields

$$
\left(\varepsilon^{2} \partial_{x}-\partial_{x}^{3}\right) \phi=z m \phi, \quad \phi_{t}=\left[z^{-1} \partial_{x}^{2}+c+u_{x}-u \partial_{x}\right] \phi
$$

which is compatible iff $m_{t}+m_{x} u+3 m u_{x}=0$ and $m_{x}=\left(u_{x x}-\varepsilon^{2} u\right)_{x}$. Letting $\varepsilon \rightarrow 0$ we obtain the system

$$
\begin{aligned}
& -\partial_{x}^{3} \phi=z m \phi, \\
& \phi_{t}=\left[z^{-1} \partial_{x}^{2}+c+u_{x}-u \partial_{x}\right] \phi,
\end{aligned}
$$

which is compatible iff $m_{t}+m_{x} u+3 m u_{x}=0$ and $m_{x}=u_{x x x}$, and hence in particular when the derivative Burgers equation holds.

Remark 2.1. Here the cubic string (2.2) on the whole real line $\mathbf{R}$ appears naturally. This is in contrast to the DP case [21] where a change of variables mapping the real line to the finite interval $(-1,1)$ is required to get rid of the $\partial_{x}$ term in the first equation of (2.1). A similar distinction holds for the ordinary string equation in the Hunter-Saxton and $\mathrm{CH}$ cases.

Remark 2.2. Hone and Wang [23] have described a different high-frequency limititing procedure taking the DP equation to (the $x$ derivative of) the integrable Vakhnenko equation $\left(u_{t}+u u_{x}\right)_{x}+u=0$ [24, 25]. It should perhaps also be mentioned that in the low-frequency limit $\epsilon \rightarrow \infty$ the substitution above directly reduces the DP equation to $u_{t}+u u_{x}=0$ [2], but this does not give a useful Lax pair.

We will return to the question of boundary conditions for (2.2) shortly. The piecewise linear solutions of the derivative Burgers equation are described by the following theorem, where we use the convention $\operatorname{sgn} 0=0$. Dots denote $\frac{\mathrm{d}}{\mathrm{d} t}$ as usual. 
Theorem 2.3. The function u given by (1.8) satisfies the derivative Burgers equation (1.5) in the sense of distributions if and only if

$$
\dot{x}_{k}=\sum_{i=1}^{n} m_{i}\left|x_{k}-x_{i}\right|, \quad \dot{m}_{k}=2 \sum_{i=1}^{n} m_{k} m_{i} \operatorname{sgn}\left(x_{i}-x_{k}\right)
$$

for $k=1, \ldots, n$.

One can assume that all $m_{k} \neq 0$, since it follows from (2.4) that any vanishing $m_{k}$ remains zero and hence never enters the solution.

The solutions in Theorem 2.3 satisfy the usual Burgers equation $u_{t}+u u_{x}=0$ (without the extra derivatives) only if $\sum_{k=1}^{n} m_{k}=0$. This case will not be considered here, since we will make the following basic assumptions throughout this paper:

$$
x_{1}<\cdots<x_{n}, \quad m_{k}>0 \text { for all } k .
$$

It can be shown that if these two assumptions hold for $t=0$, then they will continue to hold, and the solution of (2.4) will be defined, for all $t>0$. (But if $m_{k}$ 's of both signs are present, shocks may form after finite time, and then the solution will no longer have the form (1.8).) Under the assumptions (2.5), $\operatorname{sgn}\left(x_{i}-x_{k}\right)$ can be replaced by $\operatorname{sgn}(i-k)$ in (2.4).

Proposition 2.4. $M=\sum_{k=1}^{n} m_{k}$ and $M_{+}=\sum_{k=1}^{n} m_{k} x_{k}$ are constants of motion of the ODEs (2.4).

Proof. This is easily verified by a short calculation.

Since we are now in the context of piecewise linear solutions where $u$ has the form (1.8), it follows that $m=u_{x x}=2 \sum_{1}^{n} m_{k} \delta\left(x-x_{k}\right)$ is a (time-dependent) discrete measure. Consider some fixed time $t$. The wave function $\phi(x, t ; z)$ satisfying the cubic string equation (2.2) is piecewise a quadratic polynomial in $x$, since $\phi_{x x x}=0$ away from the support of $m$. At the points $x_{k},(2.2)$ implies that $\phi$ and $\phi_{x}$ are continuous while $\phi_{x x}$ has a jump of size $-2 z m_{k} \phi\left(x_{k}\right)$. These requirements completely define $\phi$ once $\phi, \phi_{x}$, and $\phi_{x x}$ are prescribed at some point.

Now let $u$ evolve according to the derivative Burgers equation; that is, let $x_{k}$ and $m_{k}$ evolve according to (2.4). Then $\phi$ evolves according to (2.3). We can postulate that

$$
\phi(x, t ; z)=1 \quad \text { for } \quad x<x_{1}(t)
$$

provided that we choose $c$ in (2.3) equal to the constant of motion $M=\sum m_{k}$; then (2.6) is compatible with the time evolution since it makes both sides of (2.3) vanish for $x<x_{1}$. Propagating (2.6) to the right gives

$$
\phi(x, t ; z)=A(t ; z) \frac{\left(x-x_{n}\right)^{2}}{2}+B(t ; z)\left(x-x_{n}\right)+C(t ; z)
$$

for $x>x_{n}(t)$, where $A, B, C$ are some explicitly computable polynomials in $z$ of degree $n$, with coefficients depending on the $x_{k}(t)$ 's and $m_{k}(t)$ 's; see (3.3) below. Inserting this into (2.3), together with $u=\sum m_{k}\left(x-x_{k}\right)=M x-M_{+}$for $x>x_{n}(t)$, gives the time evolution of these quantities:

$$
\dot{A}=0, \quad \dot{B}=M B, \quad \dot{C}=\frac{A}{z}+2 M C .
$$

We see that for $x>x_{n}$ we can impose $\phi_{x x}=2 A=0$; this condition will be preserved by the evolution in time. Together with (2.6), this amounts to $\phi$ satisfying the 
Neumann-like boundary conditions $\phi_{x}(-\infty)=\phi_{x x}(-\infty)=0, \phi_{x x}(\infty)=0$, which is how we were naturally led to the cubic string boundary value problem (1.1).

More details about the solutions of the ODEs (2.4) will be given in a separate paper devoted to the derivative Burgers equation. Here we will leave this subject after showing that $M$ is the first in a naturally appearing sequence of constants of motion, while $M_{+}$is something of an odd bird. Indeed, writing $A(z)=2 \sum_{k=1}^{n}(-z)^{k} M_{k}$ it is straightforward to derive an expression for the coefficients $M_{k}$ using (3.3), and since $A$ is constant in time, we then obtain the following result.

Theorem 2.5. The functions $M_{1}, \ldots, M_{n}$ given by

$$
M_{k}=\sum_{I \in\left(\begin{array}{c}
{[1, n]} \\
k
\end{array}\right)}\left(\prod_{i \in I} m_{i}\right)\left(\prod_{j=1}^{k-1}\left(x_{i_{j}}-x_{i_{j+1}}\right)^{2}\right) .
$$

are constants of motion for the ODEs 2.4). Here $\left(\begin{array}{c}{[1, n]} \\ k\end{array}\right)$ denotes the set of all $k$ element subsets $I=\left\{i_{1}<\cdots<i_{k}\right\}$ of the integer interval $[1, n]=\{1, \ldots, n\}$. The empty product when $k=1$ is to be interpreted as 1 , so that $M_{1}=\sum m_{k}=M$.

For example, when $n=3$ the constants of motion are

$$
\begin{aligned}
& M_{1}=m_{1}+m_{2}+m_{3}=M, \\
& M_{2}=m_{1} m_{2}\left(x_{1}-x_{2}\right)^{2}+m_{1} m_{3}\left(x_{1}-x_{3}\right)^{2}+m_{2} m_{3}\left(x_{2}-x_{3}\right)^{2}, \\
& M_{3}=m_{1} m_{2} m_{3}\left(x_{1}-x_{2}\right)^{2}\left(x_{2}-x_{3}\right)^{2},
\end{aligned}
$$

together with $M_{+}=m_{1} x_{1}+m_{2} x_{2}+m_{3} x_{3}$.

\section{The forward spectral problem}

We now turn to the spectral theory of the cubic string with Neumann-like boundary conditions (1.1). The problem makes sense for any $m(x)$ decaying sufficiently fast as $|x| \rightarrow \infty$, but we will deal exclusively with the discrete case where $m(x)=$ $2 \sum m_{k} \delta\left(x-x_{k}\right)$, and in addition we assume ordering and positivity as in (2.5). Then the eigenvalues $z$ are the zeros of the time-independent polynomial $A(z)$ defined by (2.7), so the ODEs (2.4) associated to the derivative Burgers equation induce an isospectral deformation of (1.1). If we suppress the $t$ dependence in the notation, the propagation of the wave function from left to right is described explicitly by

$$
\Phi\left(x_{k+1}^{-}\right)=L_{k} \Phi\left(x_{k}^{+}\right), \quad \Phi\left(x_{k}^{+}\right)=G_{k}(z) \Phi\left(x_{k}^{-}\right),
$$

where $\Phi=\left(\phi, \phi_{x}, \phi_{x x}\right)^{t}$ and, with $l_{k}=x_{k+1}-x_{k}$,

$$
L_{k}=\left(\begin{array}{ccc}
1 & l_{k} & l_{k}^{2} / 2 \\
0 & 1 & l_{k} \\
0 & 0 & 1
\end{array}\right), \quad G_{k}(z)=\left(\begin{array}{ccc}
1 & 0 & 0 \\
0 & 1 & 0 \\
-2 m_{k} z & 0 & 1
\end{array}\right)
$$

Hence

$$
\left(\begin{array}{c}
C(z) \\
B(z) \\
A(z)
\end{array}\right)=\Phi\left(x_{n}^{+} ; z\right)=G_{n}(z) L_{n-1} G_{n-1}(z) \ldots L_{2} G_{2}(z) L_{1} G_{1}(z)\left(\begin{array}{c}
1 \\
0 \\
0
\end{array}\right) .
$$

From this one can easily extract the coefficients of $A(z)=2 \sum_{k=1}^{n}(-z)^{k} M_{k}$ explicitly; see Theorem 2.5 above. Note also that, as $z \rightarrow 0$,

$$
\begin{aligned}
& B(z)=2 z\left(M_{+}-M x_{n}\right)+\mathrm{O}\left(z^{2}\right), \\
& C(z)=1-z \sum_{k=1}^{n} m_{k}\left(x_{n}-x_{k}\right)^{2}+\mathrm{O}\left(z^{2}\right) .
\end{aligned}
$$


Theorem 3.1. If $m_{k}>0$ for all $k$, then the spectral problem (1.1) with $m(x)=$ $2 \sum m_{k} \delta\left(x-x_{k}\right)$ has $n$ distinct nonnegative eigenvalues $z=\lambda_{k}$,

$$
0=\lambda_{0}<\lambda_{1}<\cdots<\lambda_{n-1} .
$$

Proof. It is trivial that $z=0$ is an eigenvalue, with corresponding eigenfunction $\phi=1$. The proof of the other assertions amounts to showing that the remaining eigenvalues coincide with the eigenvalues of a certain oscillatory matrix. The basic facts about oscillatory matrices are summarized in Appendix A. By (3.1), we have $\Phi\left(x_{k+1}^{+}\right)=G_{k+1}(z) L_{k} \Phi\left(x_{k}^{+}\right)$. With $\left(C_{k}, B_{k}, A_{k}\right)^{t}=\Phi\left(x_{k}^{+}\right)$, this says that

$$
\begin{aligned}
& C_{k+1}=C_{k}+l_{k} B_{k}+\frac{l_{k}^{2}}{2} A_{k}, \\
& B_{k+1}=B_{k}+l_{k} A_{k}, \\
& A_{k+1}=-2 z m_{k+1} C_{k+1}+A_{k} .
\end{aligned}
$$

The boundary conditions at $-\infty$ can be expressed as $A_{0}=B_{0}=0$. The first equation gives

$$
2 z C_{k+1}-2 z C_{k}=2 z l_{k} B_{k}+z l_{k}^{2} A_{k},
$$

which, using the second and third equations, becomes

$$
-\frac{A_{k+1}-A_{k}}{m_{k+1}}+\frac{A_{k}-A_{k-1}}{m_{k}}=2 z l_{k} \sum_{j=1}^{k-1} l_{j} A_{j}+z l_{k}^{2} A_{k} .
$$

The boundary condition at $+\infty$ is $A=A_{n}=0$, so $\mathcal{X}=\left(A_{1}, \ldots, A_{n-1}\right)^{t}$ satisfies $\mathcal{M X}=z \mathcal{L} \mathcal{X}$, where $\mathcal{M}$ is the symmetric tridiagonal matrix defined by $\mathcal{M}_{i i}=$ $m_{i}^{-1}+m_{i+1}^{-1}$ and $\mathcal{M}_{i, i-1}=\mathcal{M}_{i-1, i}=-m_{i}^{-1}$, while $\mathcal{L}$ is the lower triangular matrix with $\mathcal{L}_{i i}=l_{i}^{2}$ and $\mathcal{L}_{i j}=2 l_{i} l_{j}$ for $i>j$. $(\mathcal{M}$ and $\mathcal{L}$ are both of size $(n-1) \times(n-1)$.) An easy induction on $n$ shows that

$$
\operatorname{det} \mathcal{M}=\frac{m_{1}+m_{2}+\cdots+m_{n}}{m_{1} m_{2} \ldots m_{n}}
$$

which is positive when all $m_{k}>0$, so $\mathcal{M}^{-1}$ exists. Hence the nonzero eigenvalues of (1.1) are the reciprocals of the eigenvalues of the matrix $\mathcal{M}^{-1} \mathcal{L}$.

By Theorem A.2 we are done if we can show that $\mathcal{M}^{-1} \mathcal{L}$ is oscillatory. The star operation does not alter the determinant, since $\mathcal{M}^{*}=\mathcal{T} \mathcal{M T}$ with $\mathcal{T}=$ $\operatorname{diag}(+1,-1,+1, \ldots, \pm 1)$. Hence the determinant of $\mathcal{M}^{*}$ is positive, and so are also all its principal minors, since they are determinants of the same form. Thus $\mathcal{M}^{*}$ is positive definite, and Theorem A.5 then implies that $\mathcal{M}^{*}$ is oscillatory. Hence $\mathcal{M}^{-1}=\left(\left(\mathcal{M}^{*}\right)^{*}\right)^{-1}$ is oscillatory by Theorem A.4.

The matrix $\mathcal{L}$ is the weighted path matrix of the planar network in Figure 1 and consequently totally nonnegative by Theorem A.8 (Lindström's Lemma). Since $\mathcal{L}$ is also obviously nonsingular, $\mathcal{M}^{-1} \mathcal{L}$ is oscillatory by Theorem A.3. The proof is finished.

Next, we define the Weyl functions $W(z)$ and $Z(z)$ of the discrete cubic string with Neumann-like boundary conditions, as well as the residues $b_{k}$ and $c_{k}$ in their partial fraction decompositions. The case $M=\sum m_{k}=0$ which would need special treatment is excluded here by our standing assumption that all $m_{k}>0$. Recall that 
The inverse spectral problem for the discrete cubic string

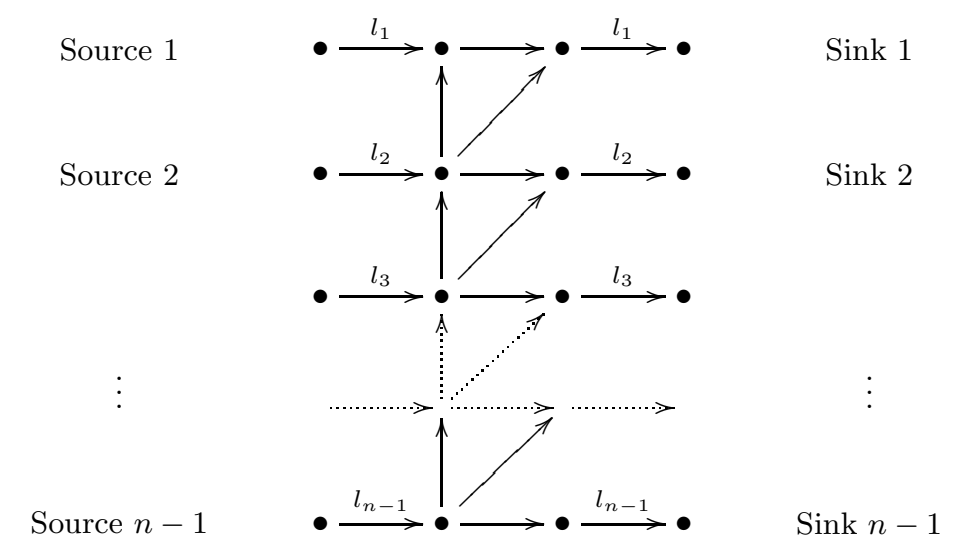

Figure 1. A planar network of order $n-1$ whose weighted path matrix is $\mathcal{L}$. Unlabelled edges have weight 1.

the zeros of $A(z)$ are $z=0$ and $z=\lambda_{k}$, and that the lowest order terms of $B(z)$ and $C(z)$ are given by (3.4).

\section{Definition 3.2. Let}

$$
\begin{aligned}
& W(z)=\frac{\phi_{x}\left(x_{n}+\right)}{\phi_{x x}\left(x_{n}+\right)}=\frac{B(z)}{A(z)}=\sum_{k=1}^{n-1} \frac{b_{k}}{z-\lambda_{k}}, \\
& Z(z)=\frac{\phi\left(x_{n}+\right)}{\phi_{x x}\left(x_{n}+\right)}=\frac{C(z)}{A(z)}=-\frac{1 / 2 M}{z}+\sum_{k=1}^{n-1} \frac{c_{k}}{z-\lambda_{k}} .
\end{aligned}
$$

Theorem 3.3. The Weyl functions satisfy the relation

$$
Z(z)+Z(-z)=W(z) W(-z) .
$$

Hence, the second Weyl function $Z$ is determined by the first Weyl function $W$ (except for the residue at $z=0$ which is determined by the constant $M$ ) through the formula

$$
c_{k}=W\left(-\lambda_{k}\right) b_{k}=-\sum_{j=1}^{n-1} \frac{b_{j} b_{k}}{\lambda_{j}+\lambda_{k}}, \quad k=1, \ldots, n-1 .
$$

Proof. Let

$$
S(z)=G_{n}(z) L_{n-1} G_{n-1}(z) \ldots L_{2} G_{2}(z) L_{1} G_{1}(z)
$$

denote the transition matrix from (3.3), whose first column is $(C, B, A)^{t}$. The $(1,3)$ entry of the identity

$$
S(-z)^{t} J S(z) J=I, \quad \text { where } \quad J=\left(\begin{array}{ccc}
0 & 0 & 1 \\
0 & -1 & 0 \\
1 & 0 & 0
\end{array}\right)
$$

reads

$$
A(-z) C(z)-B(-z) B(z)+C(-z) A(z)=0 .
$$

(Equation (3.10) is proved by noting that $X=G_{k}(z)$ and $X=L_{k}$ both satisfy $X(-z)^{-1}=(J X(z) J)^{t}$, therefore so does $X=S(z)[22$. $)$ By definition, $W=B / A$ 
and $Z=C / A$, so division by $A(z) A(-z)$ yields (3.7). The residue of (3.7) at $z=\lambda_{k}$ is 3.8 .

Remark 3.4. This proof (with trivial modifications) also works for proving the corresponding relation between the two Weyl functions for the the discrete cubic string with Dirichlet-like boundary conditions, which is used in the DP case. It is more straightforward than the proof in [22, which relied on the analogue of Theorem 3.7 below.

Theorem 3.5. If $m_{k}>0$ for all $k$, then the residues $b_{k}$ of the Weyl function $W(z)$ are all negative.

Proof. By definition $A, B$, and $C$ are all positive when $z<0$. Hence $W\left(-\lambda_{k}\right)>0$, so that $b_{k}=B\left(\lambda_{k}\right) / A^{\prime}\left(\lambda_{k}\right)$ and $c_{k}=C\left(\lambda_{k}\right) / A^{\prime}\left(\lambda_{k}\right)$ are of the same sign by (3.8); they cannot be zero, since that would imply that $A=B=C=0$ for $z=\lambda_{k}$, contradicting $\operatorname{det} S(z)=1$.

We now use induction on $n$. For $n=2$ the Weyl function is

$W(z)=\frac{B(z)}{A(z)}=\frac{-2 m_{1} l_{1} z}{-2\left(m_{1}+m_{2}\right) z+2\left(m_{1} m_{2} l_{1}^{2}\right) z^{2}}=\frac{-1 / m_{2} l_{1}}{z-\frac{m_{1}+m_{2}}{m_{1} m_{2} l_{1}^{2}}}=\frac{b_{1}}{z-\lambda_{1}}$,

so $b_{1}<0$. For $n=p>1$, if we let $m_{1} \rightarrow 0^{+}$then $W=W_{p}$ tends to the corresponding Weyl function $W_{p-1}$ for the cubic string with parameters $m_{2}, \ldots, m_{p}$ and $l_{2}, \ldots, l_{p-1}$. For the largest eigenvalue and corresponding residue, we have $\lambda_{p-1} \rightarrow+\infty$ and $b_{p-1} \rightarrow 0$, while the other eigenvalues and residues tend to the eigenvalues and (negative, by induction hypothesis) residues of $W_{p-1}$. Since the $\lambda_{k}$ 's and $b_{k}$ 's depend continuously on the parameters, the $b_{k}$ 's cannot change sign during this process, and thus they must have been negative to begin with, except perhaps for $b_{p-1}$.

To prove that $b_{p-1}<0$, note that $B$, by (3.3) and the definition of $G_{p}(z)$ and $L_{p-1}$, does not depend on $m_{p}$. By making $m_{p}$ small, we can make $\lambda_{p-1}$ as large as desired; in particular, larger in absolute value than all zeros of $B$, which except for $z=0$ are also the zeros of $W$.

Since the highest coefficients of $A$ and $B$ differ in sign, $W<0$ for all sufficiently large $z>0$. If $b_{p-1}$ were positive, then $W$ would be positive for some $z$ slightly larger than $\lambda_{p-1}$, and that would force $W$ to have a zero which is larger than $\lambda_{p-1}$, in contradiction to what we found above. Thus $b_{p-1}<0$.

Remark 3.6. Rather than using one spectrum of eigenvalues together with the corresponding residues, it is clear from (3.6) that the Weyl function $W(z)$ could instead be defined by two spectra, namely the zeros of $\phi_{x}\left(x_{n}+\right)$ and $\phi_{x x}\left(x_{n}+\right)$. Theorem 3.5 above implies that these two sets of zeros are interlacing. In this regard, and also in being determined by a single Weyl function (which is perhaps surprising), the cubic string is similar to the classical Sturm-Liouville problem and to the ordinary string.

Although it is not needed for the purposes of this paper, we also note how the Weyl functions change when the configuration $\left\{x_{k}, m_{k}\right\}_{k=1}^{n}$ of the discrete cubic string evolves in time according to the ODEs (2.4).

Theorem 3.7. The time evolution of the Weyl functions is given by

$$
\dot{W}(z)=M W(z), \quad \dot{Z}(z)=\frac{1}{z}+2 M Z(z) .
$$


Hence, the residues evolve according to

$$
b_{k}(t)=b_{k}(0) \mathrm{e}^{M t}, \quad c_{k}(t)=c_{k}(0) \mathrm{e}^{2 M t} .
$$

Proof. This follows immediately from (2.8).

\section{The inverse spectral problem}

A given discrete cubic string configuration $\left\{x_{k}, m_{k}\right\}_{k=1}^{n}$ with $x_{1}<\cdots<x_{n}$ and all $m_{k}>0$ defines, as described in the previous section, a set of spectral data consisting of the positive eigenvalues $\lambda_{1}<\cdots<\lambda_{n-1}$ together with their corresponding residues $b_{1}, \ldots, b_{n-1}<0$ and the constant of motion $M=\sum m_{k}>0$. Only the relative positions $l_{k}=x_{k+1}-x_{k}$ enter this computation, which is why $2 n-1$ is the correct number of spectral data if we wish to reconstruct the $2 n-1$ string parameters $l_{1}, \ldots, l_{n-1}$ and $m_{1}, \ldots, m_{n}$.

Let $a^{(m)}$ denote the product of the first $m$ factors in the transition matrix $S(z)$ of (3.9):

$$
\begin{aligned}
& a^{(2 k)}(z)=G_{n}(z) L_{n-1} G_{n-1}(z) L_{n-2} \ldots G_{n-k+1}(z) L_{n-k}, \\
& a^{(2 k+1)}(z)=G_{n}(z) L_{n-1} G_{n-1}(z) L_{n-2} \ldots G_{n-k+1}(z) L_{n-k} G_{n-k}(z) .
\end{aligned}
$$

The Weyl functions $W=B / A$ and $Z=C / A$ are constructed from the first column $\Phi\left(x_{n}^{+}\right)=(C, B, A)^{t}$ in $S(z)=a^{(2 n-1)}$. From the entries in the odd partial products $a^{(2 k+1)}(z)$, which are polynomials in $z$, we will show how to construct rational approximations to $W$ and $Z$ with a common denominator, and satisfying certain normalization and symmetry properties rendering the approximants unique. Conversely, given a set of spectral data, we define $W$ and $Z$ by (3.6). From the set of uniquely determined rational approximants referred to above we reconstruct all the factors $G_{j}$ and $L_{j}$ and thus solve the inverse problem.

First, we have a simple power counting lemma. Recall that $\Phi$ denotes the vector $\left(\phi, \phi_{x}, \phi_{x x}\right)$. For $0 \leq k \leq n-1$, let

$$
\left(\begin{array}{c}
p_{k}(z) \\
q_{k}(z) \\
r_{k}(z)
\end{array}\right)=\Phi\left(x_{n-k}^{-} ; z\right),
$$

so that $(C, B, A)^{t}=\Phi\left(x_{n}^{+}\right)=a^{(2 k+1)}\left(p_{k}, q_{k}, r_{k}\right)^{t}$ for any $k$.

Lemma 4.1. If we compute the polynomial degree of a matrix entry-wise, then

$$
\operatorname{deg} a^{(2 k+1)}(z)=\left(\begin{array}{ccc}
k & k-1 & k-1 \\
k & k-1 & k-1 \\
k+1 & k & k
\end{array}\right)
$$

with the proviso that for $k=0$ the degrees in the second and third columns are all zero (negative degrees counted as zero). Furthermore, $\operatorname{deg}\left(p_{k}\right)=\operatorname{deg}\left(q_{k}\right)=\operatorname{deg}\left(r_{k}\right)=$ $n-k-1$.

Proof. Since each $G_{j}(z)$ is linear in $z$, and since the leftmost factor $G_{n}(z)$ in (4.1) only affects the third row of $a^{(2 k)}(z)$, the first two rows of $a^{(2 k)}(z)$ have degree $k-1$ while the third row has degree $k$. Subsequent multiplication by $G_{n-k}(z)$ on the right gives the first claim. The second claim follows from an easy induction on $k^{\prime}=n-k$ which starts with $\left(p_{n-1}, q_{n-1}, r_{n-1}\right)=(1,0,0)$ for $k^{\prime}=1$. 
Fix $k$ and write $a(z)$ instead of $a^{(2 k+1)}(z)$ for simplicity. We now turn our attention to approximation properties of the entries of $a(z)$. These properties will later model the approximation problems required to solve the inverse problem.

Theorem 4.2. For $k$ fixed, and $z \rightarrow \infty$,

$$
\begin{aligned}
& a_{31}\left(\begin{array}{c}
Z \\
W
\end{array}\right)-\left(\begin{array}{l}
a_{11} \\
a_{21}
\end{array}\right)=\left(\begin{array}{c}
\mathrm{O}\left(z^{-1}\right) \\
\mathrm{O}(1)
\end{array}\right), \\
& a_{32}\left(\begin{array}{c}
Z \\
W
\end{array}\right)-\left(\begin{array}{l}
a_{12} \\
a_{22}
\end{array}\right)=\left(\begin{array}{c}
\mathrm{O}\left(z^{-1}\right) \\
\mathrm{O}(1)
\end{array}\right), \\
& a_{33}\left(\begin{array}{c}
Z \\
W
\end{array}\right)-\left(\begin{array}{l}
a_{13} \\
a_{23}
\end{array}\right)=\left(\begin{array}{c}
\mathrm{O}\left(z^{-2}\right) \\
\mathrm{O}\left(z^{-1}\right)
\end{array}\right),
\end{aligned}
$$

where

$$
\begin{aligned}
& \left(\begin{array}{l}
a_{11}(0) \\
a_{21}(0)
\end{array}\right)=\left(\begin{array}{l}
1 \\
0
\end{array}\right), \quad\left(\begin{array}{c}
a_{12}(0) \\
a_{22}(0)
\end{array}\right)=\left(\begin{array}{c}
\sum_{j=n-k}^{n-1} l_{j} \\
1
\end{array}\right), \\
& a_{31}(0)=a_{32}(0)=0, \quad a_{33}(0)=1 .
\end{aligned}
$$

Moreover, setting $W^{*}(z)=-W(-z)$ and $Z^{*}(z)=Z(-z)$ we have

$$
a_{3 j} Z^{*}+a_{2 j} W^{*}+a_{1 j}=\mathrm{O}\left(z^{-(k+1)}\right), \quad 1 \leq j \leq 3 .
$$

Proof. Regarding (4.4) we only give the proof for the third column of $a(z)$ to illustrate the main steps. Write $(p, q, r)$ for $\left(p_{k}, q_{k}, r_{k}\right)$. Since

$$
\left(\begin{array}{c}
Z \\
W \\
1
\end{array}\right)=\frac{1}{A}\left(\begin{array}{c}
C \\
B \\
A
\end{array}\right)=\frac{1}{A} a(z)\left(\begin{array}{c}
p \\
q \\
r
\end{array}\right)
$$

we have

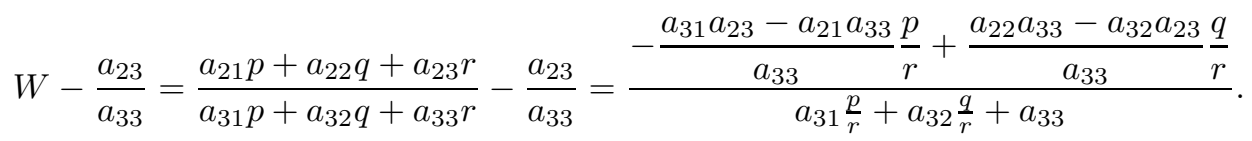

The $2 \times 2$ minors of $a(z)$ appearing here are the $(2,1)$ and $(1,1)$ entries of the adjoint matrix $\operatorname{adj}(a(z))$, which equals $a(z)^{-1}$ since each factor in $a(z)$ has determinant 1. Hence the matrix

$\operatorname{adj}(a(z))=G_{n-k}(-z) L_{n-k}^{-1} G_{n-k+1}(-z) \ldots L_{n-2}^{-1} G_{n-1}(-z) L_{n-1}^{-1} G_{n}(-z)$

has the same degree structure as in Lemma 4.1, so the minors above both have degree $k$, and so do $a_{32}$ and $a_{33}$. Moreover, $p / r$ and $q / r$ are both of order $\mathrm{O}(1)$ by Lemma 4.1, while $a_{31}$ has degree $k+1$, so the whole expression is of order $\mathrm{O}\left(z^{-(k+1)}\right)$ as claimed. The proof for $Z-a_{13} / a_{33}$ is similar, except that the appearing minors come from the first two rows of the second column of $\operatorname{adj}(a(z))$ instead, which lowers the degree one step. (4.6),

Equation (4.5) follows from (4.1) since $G_{j}(0)=I$. As for the symmetry relation

$$
a(z)^{-1}\left(\begin{array}{c}
Z \\
W \\
1
\end{array}\right)=\frac{1}{A}\left(\begin{array}{c}
p \\
q \\
r
\end{array}\right)=\left(\begin{array}{c}
\mathrm{O}\left(z^{-(k+1)}\right) \\
\mathrm{O}\left(z^{-(k+1)}\right) \\
\mathrm{O}\left(z^{-(k+1)}\right)
\end{array}\right)
$$


Like $S(z)$, the matrix $a(z)$ satisfies the property (3.10) (the proof is the same), so the left-hand side above equals $J a(-z)^{t} J(Z, W, 1)^{t}$, the rows of which give rise to (4.6) after changing $z$ to $-z$.

Proposition 4.3. For $k=0$,

$$
a_{31}^{(1)}(z)=-2 m_{n} z, \quad a_{32}^{(1)}(z)=0, \quad a_{33}^{(1)}(z)=1 .
$$

For $k=1, \ldots, n-1$,

$$
\begin{aligned}
a_{31}^{(2 k+1)}(z)=-2 z\left(\sum_{i=n-k}^{n} m_{i}\right)+\cdots+(-2 z)^{k+1}\left(\prod_{i=n+1-k}^{n} m_{i} \frac{l_{i-1}^{2}}{2}\right) m_{n-k}, \\
a_{32}^{(2 k+1)}(z)=-2 z\left(\sum_{i=n+1-k}^{n} m_{i}\left(x_{i}-x_{n-k}\right)\right) \\
+\cdots+(-2 z)^{k}\left(\prod_{i=n+2-k}^{n} m_{i} \frac{l_{i-1}^{2}}{2}\right) m_{n+1-k} l_{n-k}, \\
a_{33}^{(2 k+1)}(z)=1-2 z\left(\sum_{i=n+1-k}^{n} m_{i} \frac{\left(x_{i}-x_{n-k}\right)^{2}}{2}\right) \\
+\cdots+(-2 z)^{k}\left(\prod_{i=n+1-k}^{n} m_{i} \frac{l_{i-1}^{2}}{2}\right) .
\end{aligned}
$$

Proof. Write $G_{j}=I-2 z m_{j}(0,0,1)^{t}(1,0,0)$ in the definition (4.1) of $a^{(2 k+1)}$, and collect the lowest and highest powers of $z$.

Corollary 4.4. With [.] denoting the coefficient of the highest occurring power of $z$,

$$
\begin{aligned}
& m_{n-k}=-\frac{\left[a_{31}^{(2 k+1)}(z)\right]}{2\left[a_{33}^{(2 k+1)}(z)\right]}, \quad 0 \leq k \leq n-1, \\
& l_{n-k}=\frac{2\left[a_{33}^{(2 k+1)}(z)\right]}{\left[a_{32}^{(2 k+1)}(z)\right]}, \quad 1 \leq k \leq n-1 .
\end{aligned}
$$

Next, we show that given $W$ and $Z$, the properties in Theorem 4.2 determine $a(z)$ uniquely. Like in the DP case 22 we are dealing with a problem of simultaneous rational approximation, with approximants having a common denominator and for which the functions to be approximated are related by the quadratic constraint (3.7). In addition to the actual approximation conditions (4.4), there is also the symmetry condition (4.6) which makes the approximants unique despite the low order of the approximation condition. This type of approximation seems to be quite distinct from the cases of classical Hermite-Padé approximation known to us.

Before we proceed to the main body of the paper we need a few preparatory lemmas concerning functions $f(z)$ in the complex plane having a Laurent expansion around $z=\infty$ with finite principal part,

$$
f(z)=\sum_{j=-\infty}^{N} f_{j} z^{j} .
$$

For such functions we let $\Pi_{\geq 0}$ and $\Pi_{<0}$ denote the projection operators onto the subspaces of nonnegative and negative powers, respectively. For a fixed $\lambda$ we denote 
by $\frac{1}{z-\lambda}$ the Laurent series $z^{-1}+\lambda z^{-2}+\lambda^{2} z^{-3}+\cdots$. Finally, for $0 \leq l$, let $\Pi^{l}$ denote the projection onto $z^{-l}$. The following two lemmas are simple exercises in the algebra of Laurent series.

Lemma 4.5. Suppose $0 \leq j$ and $1 \leq l$. Then

$$
\begin{aligned}
& \Pi_{\geq 0} \frac{z^{j}}{z-\lambda}=\frac{z^{j}-\lambda^{j}}{z-\lambda}, \\
& \Pi_{<0} \frac{z^{j}}{z-\lambda}=\frac{\lambda^{j}}{z-\lambda}, \quad \Pi^{l} \frac{z^{j}}{z-\lambda}=\frac{\lambda^{j+l-1}}{z^{l}} .
\end{aligned}
$$

In particular, if $p(z)$ is a polynomial then

$$
\Pi_{\geq 0} \frac{p(z)}{z-\lambda}=\frac{p(z)-p(\lambda)}{z-\lambda}, \quad \Pi^{l} \frac{p(z)}{z-\lambda}=\frac{\lambda^{l-1} p(\lambda)}{z^{l}} .
$$

Moreover,

$$
\Pi^{0} \frac{p(z)}{z-\lambda}=\frac{p(\lambda)-p(0)}{\lambda} .
$$

Lemma 4.6. Let $\kappa \neq \lambda$ be given. Then

$$
\Pi^{l} \frac{z^{j}}{(z-\kappa)(z-\lambda)}=\frac{\kappa^{j+l-1}-\lambda^{j+l-1}}{\kappa-\lambda} z^{-l} .
$$

In particular, if $p(z)$ is a polynomial then

$$
\Pi^{l} \frac{p(z)}{(z-\kappa)(z-\lambda)}=\frac{\kappa^{l-1} p(\kappa)-\lambda^{l-1} p(\lambda)}{\kappa-\lambda} z^{-l} .
$$

Moreover,

$$
\Pi^{0} \frac{p(z)}{(z-\kappa)(z-\lambda)}=\frac{1}{\kappa-\lambda}\left[\frac{p(\kappa)-p(0)}{\kappa}-\frac{p(\lambda)-p(0)}{\lambda}\right]
$$

We will introduce three types of approximation problem, each type specifically referring to the pertinent column of $a(z)$ as in Theorem 4.2. The following setup is common to all three cases.

Definition 4.7. Let $\left\{\lambda_{k}, b_{k}, M\right\}$ be arbitrary numbers satisfying the constraints

$$
0<\lambda_{1}<\lambda_{2}<\cdots<\lambda_{n-1}, \quad b_{1}, \ldots, b_{n-1}<0, \quad M>0 .
$$

Define (signed) measures

$$
\mathrm{d} \mu(\lambda)=\sum_{k=1}^{n-1} b_{k} \delta\left(\lambda-\lambda_{k}\right), \quad \mathrm{d} \nu(\lambda)=-\frac{1}{2 M} \delta(\lambda)+\sum_{k=1}^{n-1} c_{k} \delta\left(\lambda-\lambda_{k}\right),
$$

where the $c_{k}$ 's are given by (3.8), which amounts to

$$
-\int f(\lambda) \mathrm{d} \nu(\lambda)=\frac{f(0)}{2 M}+\iint \frac{f(\lambda)}{\kappa+\lambda} \mathrm{d} \mu(\kappa) \mathrm{d} \mu(\lambda)
$$

for any function $f$. Define the two functions

$$
\begin{aligned}
& W(z)=\int \frac{\mathrm{d} \mu(\lambda)}{z-\lambda}=\sum_{k=1}^{n-1} \frac{b_{k}}{z-\lambda_{k}}, \\
& Z(z)=\int \frac{\mathrm{d} \nu(\lambda)}{z-\lambda}=-\frac{1 / 2 M}{z}+\sum_{k=1}^{n-1} \frac{c_{k}}{z-\lambda_{k}},
\end{aligned}
$$


and let

$W^{*}(z)=-W(-z)=\int \frac{\mathrm{d} \mu(\lambda)}{z+\lambda}, \quad Z^{*}(z)=Z(-z)=-\int \frac{\mathrm{d} \nu(\lambda)}{z+\lambda}$.

(Note that $Z(z)$ is determined by $W(z)$ and $M$, and that $W$ and $Z$ satisfy (3.7).) Finally, let

$$
\beta_{j}=\int \lambda^{j} \mathrm{~d} \mu(\lambda), \quad I_{i j}=I_{j i}=\iint \frac{\kappa^{i} \lambda^{j}}{\kappa+\lambda} \mathrm{d} \mu(\kappa) \mathrm{d} \mu(\lambda) .
$$

Definition 4.8. Let $\mathcal{A}_{0}=\mathcal{B}_{0}=\mathcal{C}_{0}=\mathcal{D}_{0}=1, \mathcal{A}_{1}=I_{00}+\frac{1}{2 M}, \mathcal{D}_{1}^{\prime}=\beta_{0}$, and

$$
\begin{aligned}
& \mathcal{A}_{k}=\left|\begin{array}{cccc}
I_{00}+\frac{1}{2 M} & I_{01} & \cdots & I_{0, k-1} \\
I_{10} & I_{11} & \cdots & I_{1, k-1} \\
I_{20} & I_{21} & \cdots & I_{2, k-1} \\
\vdots & \vdots & & \vdots \\
I_{k-1,0} & I_{k-1,1} & \cdots & I_{k-1, k-1}
\end{array}\right|, \quad 2 \leq k, \\
& \mathcal{B}_{k}=\left|\begin{array}{cccc}
I_{00} & I_{01} & \cdots & I_{0, k-1} \\
I_{10} & I_{11} & \cdots & I_{1, k-1} \\
\vdots & \vdots & & \vdots \\
I_{k-1,0} & I_{k-1,1} & \cdots & I_{k-1, k-1}
\end{array}\right|, \quad 1 \leq k, \\
& \mathcal{C}_{k}=\left|\begin{array}{cccc}
I_{11} & I_{12} & \cdots & I_{1 k} \\
I_{21} & I_{22} & \cdots & I_{2 k} \\
\vdots & \vdots & & \vdots \\
I_{k 1} & I_{k 2} & \cdots & I_{k k}
\end{array}\right|, \quad 1 \leq k, \\
& \mathcal{D}_{k}=\left|\begin{array}{cccc}
I_{10} & I_{11} & \cdots & I_{1, k-1} \\
I_{20} & I_{21} & \cdots & I_{2, k-1} \\
\vdots & \vdots & & \vdots \\
I_{k 0} & I_{k 1} & \cdots & I_{k, k-1}
\end{array}\right|, \quad 1 \leq k, \quad 2 \leq k . \\
& \mathcal{D}_{k}^{\prime}=\left|\begin{array}{cccc}
\beta_{0} & I_{10} & \cdots & I_{1, k-2} \\
\beta_{1} & I_{20} & \cdots & I_{2, k-2} \\
\vdots & \vdots & & \vdots \\
\beta_{k-1} & I_{k 0} & \cdots & I_{k, k-2}
\end{array}\right|, \quad 2
\end{aligned}
$$

In all these cases, the index $k$ agrees with the size $k \times k$ of the determinant. Note also that $\mathcal{A}_{k}=\mathcal{B}_{k}+\frac{1}{2 M} \mathcal{C}_{k-1}$ for $k \geq 1$.

Theorem 4.9 (Type III approximation problem). Fix $1 \leq k \leq n-1$. There are unique polynomials $Q(z), P(z), \widehat{P}(z)$ of degree $k, k-1, k-1$, respectively, satisfying the following properties:

(i) Approximation:

$$
Q\left(\begin{array}{c}
Z \\
W
\end{array}\right)-\left(\begin{array}{c}
\widehat{P} \\
P
\end{array}\right)=\mathrm{O}\left(z^{-1}\right), \quad \text { as } z \rightarrow \infty .
$$

(ii) Symmetry:

$$
\widehat{P}+P W^{*}+Q Z^{*}=\mathrm{O}\left(z^{-(k+1)}\right), \quad \text { as } z \rightarrow \infty .
$$


(iii) Normalization: $Q(0)=1$.

The coefficients of $Q(z)=1+\sum_{j=1}^{k} q_{j} z^{j}$ are given by the $k \times k$ linear system

$$
\left(\begin{array}{cccc}
I_{01} & I_{02} & \cdots & I_{0 k} \\
I_{11} & I_{12} & \cdots & I_{1 k} \\
\vdots & & & \vdots \\
I_{k-1,1} & I_{k-1,2} & \cdots & I_{k-1, k}
\end{array}\right)\left(\begin{array}{c}
q_{1} \\
q_{2} \\
\vdots \\
q_{k}
\end{array}\right)=-\left(\begin{array}{c}
I_{00}+\frac{1}{2 M} \\
I_{10} \\
\vdots \\
I_{k-1,0}
\end{array}\right) .
$$

The polynomials $P(z)$ and $\widehat{P}(z)$ are determined from $Q(z)$ through (4.26) below.

Proof. Upon applying $\Pi_{\geq 0}$ and Lemma 4.5 to (4.23) we obtain

$$
\begin{aligned}
& P(z)=\Pi_{\geq 0}(W Q)=\int \frac{Q(z)-Q(\lambda)}{z-\lambda} \mathrm{d} \mu(\lambda), \\
& \widehat{P}(z)=\Pi_{\geq 0}(Z Q)=\int \frac{Q(z)-Q(\lambda)}{z-\lambda} \mathrm{d} \nu(\lambda),
\end{aligned}
$$

so that $P$ and $\widehat{P}$ are uniquely determined once we know $Q$. Inserting this into (4.24) yields

$$
\Pi_{\geq 0}(Z Q)+\Pi_{\geq 0}(W Q) W^{*}+Q Z^{*}=\mathrm{O}\left(z^{-(k+1)}\right) .
$$

Applying $\Pi_{\geq 0}$ to the left-hand side yields $\Pi_{\geq 0}\left(\left(Z+Z^{*}+W W^{*}\right) Q\right)$, which is zero by (3.7). Thus, the nonnegative powers in (4.27) vanish automatically, while the vanishing of the powers $z^{-1}, \ldots, z^{-k}$ is the condition that will determine the $k$ unknown coefficients in $Q(z)$. Define the Hankel and Toeplitz operators

$$
H_{f}=\Pi_{<0} \circ M_{f} \circ \Pi_{\geq 0}, \quad T_{f}=\Pi_{\geq 0} \circ M_{f} \circ \Pi_{\geq 0},
$$

where $M_{f}$ denotes multiplication by the function $f$. Applying $\Pi_{<0}$ to the left-hand side of (4.27) yields $H_{Z^{*}} Q+H_{W^{*}} T_{W} Q$, so that $Q$ is determined by the linear system

$$
\left(H_{Z^{*}}+H_{W^{*}} T_{W}\right) Q=\mathrm{O}\left(z^{-(k+1)}\right) \text {. }
$$

If we restrict the operators to the finite-dimensional subspaces spanned by $\left\{1, z, \ldots, z^{k}\right\}$ and $\left\{z^{-1}, \ldots, z^{-k}\right\}$ we obtain $k$ equations

$$
\Pi^{l}\left(H_{Z^{*}}+H_{W^{*}} T_{W}\right) Q=0, \quad 1 \leq l \leq k .
$$

With the help of Lemma 4.5 this can be rewritten as

$$
\Pi^{l}\left(-\int \frac{Q(z)}{z+\lambda} \mathrm{d} \nu(\lambda)+\iint \frac{Q(z)-Q(\kappa)}{(z-\kappa)(z+\lambda)} \mathrm{d} \mu(\kappa) \mathrm{d} \mu(\lambda)\right)=0,
$$

which according to the relation (4.14) between the measures $\mathrm{d} \mu$ and $\mathrm{d} \nu$ equals

$$
\Pi^{l}\left(\frac{Q(z) / z}{2 M}+\iint\left(\frac{Q(z) /(z+\lambda)}{\kappa+\lambda}+\frac{Q(z)-Q(\kappa)}{(z-\kappa)(z+\lambda)}\right) \mathrm{d} \mu(\kappa) \mathrm{d} \mu(\lambda)\right)=0 .
$$

Finally, upon executing the projection $\Pi^{l}$ for $1 \leq l \leq k$ using formulas from Lemmas 4.5 and 4.6, we obtain

$$
\begin{aligned}
& \frac{Q(0)}{2 M}+\iint \frac{Q(\kappa)}{\kappa+\lambda} \mathrm{d} \mu(\kappa) \mathrm{d} \mu(\lambda)=0, \\
& \iint \frac{(\lambda)^{l-1} Q(\kappa)}{\kappa+\lambda} \mathrm{d} \mu(\kappa) \mathrm{d} \mu(\lambda)=0, \quad 2 \leq l \leq k .
\end{aligned}
$$


When $Q$ is represented by the column vector $\left(1, q_{1}, \ldots, q_{k}\right)^{t}$ this takes the form (4.25). The determinant of the system (4.25) is $\mathcal{D}_{k}$ of Definition 4.8, which is positive by Lemma B.1 in Appendix B. Thus the solution is unique.

Definition 4.10. Denote the results of the $k$ th approximation problem of type III by $Q_{3 k}, P_{3 k}, \widehat{P}_{3 k}$.

Corollary 4.11. The highest order coefficient of $Q_{3 k}(z)$ is $\left[Q_{3 k}\right]=(-1)^{k} \mathcal{A}_{k} / \mathcal{D}_{k}$.

Theorem 4.12 (Type II approximation problem). Fix $1 \leq k \leq n-1$. There are unique polynomials $Q(z), P(z), \widehat{P}(z)$ of degree $k, k-1, k-1$, respectively, satisfying the following properties:

(i) Approximation:

$$
Q\left(\begin{array}{c}
Z \\
W
\end{array}\right)-\left(\begin{array}{c}
\widehat{P} \\
P
\end{array}\right)=\left(\begin{array}{c}
\mathrm{O}\left(z^{-1}\right) \\
\mathrm{O}(1)
\end{array}\right), \quad \text { as } z \rightarrow \infty
$$

(ii) Symmetry:

$$
\widehat{P}+P W^{*}+Q Z^{*}=\mathrm{O}\left(z^{-(k+1)}\right), \quad \text { as } z \rightarrow \infty .
$$

(iii) Normalization: $Q(0)=0, P(0)=1$.

The coefficients of $Q(z)=\sum_{j=1}^{k} q_{j} z^{j}$ are given by the $k \times k$ linear system

$$
\left(\begin{array}{cccc}
I_{10} & I_{11} & \cdots & I_{1, k-1} \\
I_{20} & I_{21} & \cdots & I_{2, k-1} \\
\vdots & & & \vdots \\
I_{k 0} & I_{k 1} & \cdots & I_{k, k-1}
\end{array}\right)\left(\begin{array}{c}
q_{1} \\
q_{2} \\
\vdots \\
q_{k}
\end{array}\right)=\left(\begin{array}{c}
\beta_{0} \\
\beta_{1} \\
\vdots \\
\beta_{k-1}
\end{array}\right) .
$$

The polynomials $P(z)$ and $\widehat{P}(z)$ are determined from $Q(z)$ through 4.37) below.

Proof. The proof proceeds in a similar way to the proof of Theorem 4.9 We therefore only indicate the steps which are new to the present case. First, from the normalization condition we get

$$
\begin{aligned}
& P(z)=1+\Pi_{>0}(W Q)=\Pi_{\geq 0}(W Q)+1-\Pi^{0}(W Q), \\
& \widehat{P}(z)=\Pi_{\geq 0}(Z Q)=\int \frac{Q(z)-Q(\lambda)}{z-\lambda} \mathrm{d} \nu(\lambda) .
\end{aligned}
$$

Inserting this into (4.35) yields

$\Pi_{\geq 0}(Z Q)+\Pi_{\geq 0}(W Q) W^{*}+Q Z^{*}+\left(1-\Pi^{0}(W Q)\right) W^{*}=\mathrm{O}\left(z^{-(k+1)}\right)$.

Applying $\Pi_{\geq 0}$ to the left-hand side yields $\Pi_{\geq 0}\left(\left(Z+Z^{*}+W W^{*}\right) Q\right)$, which is zero by (3.7). Thus, the nonnegative powers in (4.38) vanish automatically, while the vanishing of the powers $z^{-1}, \ldots, z^{-k}$ is the condition that will determine the $k$ unknown coefficients in $Q(z)$. With the help of the Hankel and Toeplitz operators defined in the previous proof we find that $Q$ is determined by the linear system

$$
\left(H_{Z^{*}}+H_{W^{*}} T_{W}\right) Q+\left(1-\Pi^{0}(W Q)\right) W^{*}=\mathrm{O}\left(z^{-(k+1)}\right),
$$

where we have used that $W^{*}=\mathrm{O}(1 / z)$ according to its definition. If we restrict the operators to the finite-dimensional subspaces spanned by $\left\{1, z, \ldots, z^{k}\right\}$ and $\left\{z^{-1}, \ldots, z^{-k}\right\}$ we obtain the $k$ equations

$\Pi^{l}\left(\left(H_{Z^{*}}+H_{W^{*}} T_{W}\right) Q+\left(1-\Pi^{0}(W Q)\right) W^{*}\right)=0, \quad 1 \leq l \leq k$. 
With the help of Lemma 4.6 and the relation (4.14) between the measures $\mathrm{d} \mu$ and $\mathrm{d} \nu$ we obtain

$$
\iint \frac{\lambda^{l} Q(\kappa)}{\kappa(\kappa+\lambda)} \mathrm{d} \mu(\kappa) \mathrm{d} \mu(\lambda)=\int(\lambda)^{l-1} \mathrm{~d} \mu(\lambda), \quad 1 \leq l \leq k .
$$

When $Q$ is represented by the column vector $\left(q_{1}, \ldots, q_{k}\right)^{t}$ this gives the system (4.36), which has the same determinant $\mathcal{D}_{k}>0$ as (4.25). Hence, the solution is again unique.

Definition 4.13. Denote the results of the $k$ th approximation problem of type II by $Q_{3 k+1}, P_{3 k+1}, \widehat{P}_{3 k+1}$.

Corollary 4.14. The highest order coefficient of $Q_{3 k+1}(z)$ is $\left[Q_{3 k+1}\right]=$ $(-1)^{k-1} \mathcal{D}_{k}^{\prime} / \mathcal{D}_{k}$.

Theorem 4.15 (Type I approximation problem). Let $0 \leq k \leq n-1$. Then there are unique polynomials $Q(z), P(z), \widehat{P}(z)$ of degree $k+1, k, k$, respectively, satisfying the following properties:

(i) Approximation:

$$
Q\left(\begin{array}{c}
Z \\
W
\end{array}\right)-\left(\begin{array}{c}
\widehat{P} \\
P
\end{array}\right)=\left(\begin{array}{c}
\mathrm{O}\left(z^{-1}\right) \\
\mathrm{O}(1)
\end{array}\right), \quad \text { as } z \rightarrow \infty
$$

(ii) Symmetry:

$$
\widehat{P}+P W^{*}+Q Z^{*}=\mathrm{O}\left(z^{-(k+1)}\right), \quad \text { as } z \rightarrow \infty .
$$

(iii) Normalization: $Q(0)=0, P(0)=0, \widehat{P}(0)=1$.

The coefficients of $Q(z)=\sum_{j=1}^{k+1} q_{j} z^{j}$ are given by the $(k+1) \times(k+1)$ linear system

$$
\left(\begin{array}{cccc}
I_{00}+\frac{1}{2 M} & I_{01} & \cdots & I_{0 k} \\
I_{10} & I_{11} & \cdots & I_{1 k} \\
I_{20} & I_{21} & \cdots & I_{2 k} \\
\vdots & & & \vdots \\
I_{k 0} & I_{k 1} & \cdots & I_{k k}
\end{array}\right)\left(\begin{array}{c}
q_{1} \\
q_{2} \\
q_{3} \\
\vdots \\
q_{k+1}
\end{array}\right)=-\left(\begin{array}{c}
1 \\
0 \\
0 \\
\vdots \\
0
\end{array}\right) .
$$

The polynomials $P(z)$ and $\widehat{P}(z)$ are determined from $Q(z)$ through 4.45) below.

Proof. From the normalization and approximation conditions we get

$$
\begin{aligned}
& P(z)=\Pi_{>0}(W Q)=\Pi_{\geq 0}(W Q)-\Pi^{0}(W Q), \\
& \widehat{P}(z)=\Pi_{\geq 0}(Z Q)=\int \frac{Q(z)-Q(\lambda)}{z-\lambda} \mathrm{d} \nu(\lambda) .
\end{aligned}
$$

Inserting this into (4.43) yields

$\Pi_{\geq 0}(Z Q)+\Pi_{\geq 0}(W Q) W^{*}+Q Z^{*}-\Pi^{0}(W Q) W^{*}=\mathrm{O}\left(z^{-(k+1)}\right)$.

Applying $\Pi_{\geq 0}$ to the left-hand side yields $\Pi_{\geq 0}\left(\left(Z+Z^{*}+W W^{*}\right) Q\right)$, which is zero by (3.7). Thus, the nonnegative powers in (4.46) vanish automatically, while the vanishing of the powers $z^{-1}, \ldots, z^{-k}$ will give $k$ conditions on the $k+1$ unknown coefficients in $Q(z)$, which together with the normalization condition $\widehat{P}(0)=1$ is the 
required number of conditions. Symbolically, with the help of the Hankel and Toeplitz operators defined earlier, we find that $Q$ is determined by the linear system

$$
\begin{aligned}
& \left(H_{Z^{*}}+H_{W^{*}} T_{W}\right) Q-\Pi^{0}(W Q) W^{*}=\mathrm{O}\left(z^{-(k+1)}\right), \\
& \Pi^{0}(Z Q)=1 .
\end{aligned}
$$

If we restrict the operators to the finite-dimensional subspaces spanned by $\left\{z, \ldots, z^{k+1}\right\}$ and $\left\{z^{-1}, \ldots, z^{-k}\right\}$, then the $k$ equations alluded to earlier are

$\Pi^{l}\left(\left(H_{Z^{*}}+H_{W^{*}} T_{W}\right) Q-\Pi^{0}(W Q) W^{*}\right)=0, \quad 1 \leq l \leq k$.

Likewise, Lemma 4.6 and the relation (4.14) between the measures $\mathrm{d} \mu$ and $\mathrm{d} \nu$ imply that the complete set of equations determining $Q$ reads

$$
\begin{aligned}
& \int \frac{Q(\lambda)}{\lambda} \mathrm{d} \nu(\lambda)=1, \\
& \iint \frac{\lambda^{l} Q(\kappa)}{\kappa(\kappa+\lambda)} \mathrm{d} \mu(\kappa) \mathrm{d} \mu(\lambda)=0, \quad 1 \leq l \leq k .
\end{aligned}
$$

When $Q$ is represented by the column vector $\left(q_{1}, \ldots, q_{k+1}\right)^{t}$ this gives (4.44). The determinant of the matrix of coefficients is $\mathcal{A}_{k+1}=\mathcal{B}_{k+1}+\frac{1}{2 M} \mathcal{C}_{k}$ in the notation of Definition 4.8. Following the methods used in [22, Lemma 4.10] one can establish Heine-type formulas for both determinants in this sum, which show that both are strictly positive provided $k<n-1$; see Lemmas B.2 and B.3 in Appendix B. From these formulas one also concludes that when $k=n-1$ the first determinant is zero, since the measure $\mathrm{d} \mu(\lambda)$ is supported on $n-1$ points, while the second remains strictly positive. This proves that the system (4.44) has a unique solution for the claimed range of $k$.

Definition 4.16. Denote the results of the $k$ th approximation problem of type I by $Q_{3 k+2}, P_{3 k+2}, \widehat{P}_{3 k+2}$.

Corollary 4.17. The highest order coefficient of $Q_{3 k+2}(z)$ is $\left[Q_{3 k+2}\right]=$ $(-1)^{k+1} \mathcal{C}_{k} / \mathcal{A}_{k}$.

The last approximation problem of type I has a particular significance.

Corollary 4.18. For $k=n-1$, the approximation problem of type I gives

$$
Q_{3 n-1}(z)=-2 M z \prod_{j=1}^{n-1}\left(1-\frac{z}{\lambda_{j}}\right) .
$$

Moreover, the approximation is exact:

$$
Z(z)=\frac{\widehat{P}_{3 n-1}(z)}{Q_{3 n-1}(z)}, \quad W(z)=\frac{P_{3 n-1}(z)}{Q_{3 n-1}(z)} .
$$

Proof. We write the conditions (4.49) that $Q_{3 n-1}$ has to satisfy:

$$
\begin{aligned}
& \int \frac{Q_{3 n-1}(\lambda)}{\lambda} \mathrm{d} \nu(\lambda)=1, \\
& \iint \frac{\lambda^{i} Q_{3 n-1}(\kappa)}{\kappa(\kappa+\lambda)} \mathrm{d} \mu(\kappa) \mathrm{d} \mu(\lambda)=0, \quad 1 \leq i \leq n-1 .
\end{aligned}
$$


We claim that $Q_{3 n-1}$ has to vanish on the support of the measure $\mathrm{d} \mu$. We recall that $Q_{3 n-1}$ is a polynomial of degree $n$ with one root at $\lambda=0$ by the definition of the approximation problem of type I. Thus the claim is that the remaining roots are precisely the $\lambda_{j}$ occuring in (4.13). To prove that, we write explicitly the second condition,

$$
\sum_{j=1}^{n-1}\left(\int \frac{\lambda^{i}}{\lambda+\lambda_{j}} \mathrm{~d} \mu(\lambda)\right)\left(\frac{Q_{3 n-1}\left(\lambda_{j}\right) b_{j}}{\lambda_{j}}\right)=0, \quad 1 \leq i \leq n-1 .
$$

It is straightforward to establish a multiple integral formula of Heine type for the matrix of coefficients, from which one concludes that the matrix is nonsingular; see (B.13) in Appendix B. This implies that $Q_{3 n-1}\left(\lambda_{j}\right)=0$ for $1 \leq j \leq n-1$, which proves the claim. Moreover, the definition of the measure $\mathrm{d} \nu$ and the first condition in (4.52) imply that the coefficient of the linear term of $Q_{3 n-1}$ is $-2 M$, which proves the product formula for $Q_{3 n-1}$. To prove that the $k=n-1$ approximation of type I is exact we show that

$$
\Pi_{<0}\left(Q_{3 n-1}\left(\begin{array}{c}
Z \\
W
\end{array}\right)-\left(\begin{array}{c}
\widehat{P}_{3 n-1} \\
P_{3 n-1}
\end{array}\right)\right)=0 .
$$

This follows from Lemma 4.5, since for example

$$
\Pi_{<0}\left(Q_{3 n-1} W\right)(z)=\int \frac{Q_{3 n-1}(z)}{z-\lambda} \mathrm{d} \mu(\lambda)=\int \frac{Q_{3 n-1}(\lambda)}{z-\lambda} \mathrm{d} \mu(\lambda)=0
$$

because $Q_{3 n-1}$ vanishes on the support of $\mathrm{d} \mu$. Likewise,

$$
\Pi^{0}\left(Q_{3 n-1} W\right)=\Pi^{0} \int \frac{Q_{3 n-1}(z)}{z-\lambda} \mathrm{d} \mu(\lambda)=\int \frac{Q_{3 n-1}(\lambda)}{\lambda} \mathrm{d} \mu(\lambda)=0 .
$$

The rest follows from the definition of the approximation problem of type I.

We can now present the solution of the uniqueness part of the inverse problem.

Theorem 4.19. Let

$$
0<\lambda_{1}<\lambda_{2}<\cdots<\lambda_{n-1}, \quad b_{1}, \ldots, b_{n-1}<0, \quad M>0
$$

be the spectral data (as described in Section [3) of a cubic string $\left\{m_{k}, x_{k}\right\}_{k=1}^{n}$ with $m_{k}>0$ and $x_{1}<\cdots<x_{n}$. Then the string data can be recovered uniquely from the spectral data, up to a translation along the $x$ axis, from the determinantal formulas

$$
m_{n-k}=\frac{\mathcal{C}_{k} \mathcal{D}_{k}}{2 \mathcal{A}_{k+1} \mathcal{A}_{k}}, \quad l_{n-k}=-\frac{2 \mathcal{A}_{k}}{\mathcal{D}_{k}^{\prime}},
$$

where $l_{j}=x_{j+1}-x_{j}$. (See Definitions 4.7 and 4.8 for notation.)

Proof. Equations (4.54) follow from Corollaries 4.11, 4.14, 4.17 and equations 4.11) and (4.12).

To show the existence part (that any numbers satisfying the constraints (4.53) really are spectral data of some cubic string), we need the following theorem, whose proof will be published elsewhere. Note that the orthogonal polynomials occurring in the description of continued fractions of Stieltjes type satisfy three-term recurrence relations. It is therefore gratifying to know that the polynomials occurring in the approximation problems for the cubic string satisfy four-term recurrence relations. 
Theorem 4.20. Consider the same setup as in Definition 4.7 (numbers $\left\{\lambda_{k}, b_{k}, M\right\}$ given), and let the polynomials $Q_{j}(z), P_{j}(z), \widehat{P}_{j}(z)$ be defined (for $j \geq 2$ ) by the unique solutions of the approximation problems of type I, II and III, as in Definitions 4.10, 4.13 and 4.16. Define $l_{j}$ and $m_{j}$ from the numbers $\left\{\lambda_{k}, b_{k}, M\right\}$ via (4.54), and consider the recurrence relations

$X_{3 k}=\frac{1}{2} l_{n-k}^{2} X_{3 k-1}+l_{n-k} X_{3 k-2}+X_{3 k-3}, \quad 1 \leq k \leq n-1$,

$X_{3 k+1}=l_{n-k} X_{3 k-1}+X_{3 k-2}, \quad 1 \leq k \leq n-1$,

$X_{3 k+2}=-2 z m_{n-k} X_{3 k}+X_{3 k-1}, \quad 0 \leq k \leq n-1$.

Then with initial conditions $\left(X_{-1}, X_{0}, X_{1}\right)=(1,0,0)$ the solution (for $\left.j \geq 2\right)$ is $X_{j}=\widehat{P}_{j}$, when $\left(X_{-1}, X_{0}, X_{1}\right)=(0,1,0)$ the solution is $X_{j}=Q_{j}$, and when $\left(X_{-1}, X_{0}, X_{1}\right)=(0,0,1)$ the solution is $X_{j}=P_{j}$.

Theorem 4.21. Given any numbers $\left\{\lambda_{k}, b_{k}, M\right\}$ satisfying the constraints 4.53), the formulas (4.54) define a cubic string with these numbers as spectral data.

Proof. Note first that all $m_{j}$ and $l_{j}$ defined by (4.54) are strictly positive. This follows since $\mathcal{D}_{k}^{\prime}<0$ according to (B.5) and our definition of $\mathrm{d} \mu(\lambda)$ as a negative measure, while the remaining determinants are strictly positive. This means that we have a valid cubic string, but we do not know yet whether its spectral data $\left\{\widetilde{\lambda}_{k}, \widetilde{b}_{k}, \widetilde{M}\right\}$ coincide with the numbers $\left\{\lambda_{k}, b_{k}, M\right\}$ that we started with. However, plugging $\left\{\widetilde{\lambda}_{k}, \widetilde{b}_{k}, \widetilde{M}\right\}$ into (4.54) recovers the same string data $\left\{m_{j}, l_{j}\right\}$ by uniqueness (Theorem 4.19). Thus, applying Theorem 4.20 to the set of numbers $\left\{\lambda_{k}, b_{k}, M\right\}$ produces the same polynomials $Q_{j}, P_{j}, \widehat{P}_{j}$ as one would get with $\left\{\widetilde{\lambda}_{k}, \widetilde{b}_{k}, \widetilde{M}\right\}$, since the string data are the same in both cases. And according to Corollary 4.18, both $M$ and $W(z)$ are uniquely determined from these polynomials. Thus $\widetilde{\lambda}_{k}=\lambda_{k}, \widetilde{b}_{k}=b_{k}$, and $\widetilde{M}=M$, which is what we needed to show.

\section{Acknowledgments}

JK wishes to acknowledge the support received from the National Science and Engineering Research Council of Canada (NSERC) in the form of the Undergraduate Student Research Award. HL is grateful to the Institut Mittag-Leffler, Djursholm, Sweden, for its hospitality during the Fall 2005 program "Wave Motion". The research of JS is supported by NSERC.

\section{Appendix A. A collection of facts about totally nonnegative and oscillatory matrices}

We proved that the spectrum of the Neumann-like discrete cubic string (1.1) is positive and simple by rewriting it as a matrix eigenvalue problem with an oscillatory matrix. Below, the reader will find some relevant definitions and theorems from the vast area of totally nonnegative matrices; for details see the book by Gantmacher and Krein [26] and the excellent survey by Fomin and Zelevinsky [27.

Definition A.1. A square matrix is totally nonnegative (TN) if all its minors are nonnegative. It is totally positive $(T P)$ if all its minors are positive. It is oscillatory if it is TN and some power of it is TP. 
Theorem A.2. All eigenvalues of a TP matrix are positive and of algebraic multiplicity one, and likewise for oscillatory matrices. All eigenvalues of a TN matrix are nonnegative, but in general of arbitrary multiplicity.

Theorem A.3. The product of an oscillatory matrix and a nonsingular TN matrix is oscillatory.

Theorem A.4 (Star operation). If $A=\left(a_{i j}\right)$ is oscillatory, then $\left(A^{*}\right)^{-1}$ is oscillatory, where $A^{*}=\left((-1)^{i+j} a_{i j}\right)$.

Theorem A.5. A symmetric tridiagonal matrix is oscillatory if and only if it is positive definite and has positive off-diagonal entries.

Definition A.6. A planar network $(\Gamma, \omega)$ of order $n$ is an acyclic, planar directed graph $\Gamma$ with arrows going from left to right, with $n$ sources (vertices with outgoing arrows only) on the left side, and with $n$ sinks (vertices with incoming arrows only) on the right side. The sources and sinks are numbered 1 to $n$ from top to bottom. All other vertices have at least one arrow coming in and at least one arrow going out. Each edge $e$ of the graph $\Gamma$ is assigned a scalar weight $\omega(e)$.

Definition A.7. Given a planar network $\Gamma$ of order $n$, the weight of a directed path in $\Gamma$ is the product of all the weights of the edges of that path. The weighted path matrix $A(\Gamma, \omega)$ is the $n \times n$ matrix whose $(i, j)$ entry is the sum of the weights of the possible paths from source $i$ to sink $j$.

The following famous theorem was discovered independently by Lindström, Karlin-McGregor, and Gessel-Viennot.

Theorem A.8. Let $I$ and $J$ be subsets of $\{1, \ldots, n\}$ with the same cardinality. The minor

$$
\Delta_{I, J}(A)=\operatorname{det}\left(a_{i j}\right)_{i \in I, j \in J}
$$

of the weighted path matrix $A(\Gamma, \omega)$ of a planar network is equal to the sum of the weights of all possible families of vertex-disjoint paths connecting the sources labelled by $I$ to the sinks labelled by $J$. (The weight of a family of paths is defined as the product of the weights of the individual paths.)

Corollary A.9. If all weights $\omega$ are nonnegative, then the weighted path matrix is $T N$.

\section{Appendix B. Heine-like determinant formulas}

The following was proved in [22], where it appeared as Lemma 4.10.

Lemma B.1. Suppose $\mu$ is a measure on $\mathbf{R}$ such that the integrals

$$
\beta_{a}=\int x^{a} \mathrm{~d} \mu(x), \quad I_{a b}=\iint \frac{x^{a} y^{b}}{x+y} \mathrm{~d} \mu(x) \mathrm{d} \mu(y)
$$

are finite. Let

$$
\begin{aligned}
& u_{k}=\frac{1}{k !} \int_{\mathbf{R}^{k}} \frac{\Delta(x)^{2}}{\Gamma(x)} \mathrm{d} \mu^{k}(x), \\
& v_{k}=\frac{1}{k !} \int_{\mathbf{R}^{k}} \frac{\Delta(x)^{2}}{\Gamma(x)} x_{1} x_{2} \ldots x_{k} \mathrm{~d} \mu^{k}(x),
\end{aligned}
$$


where

$$
\begin{aligned}
& \Delta(x)=\Delta\left(x_{1}, \ldots, x_{k}\right)=\prod_{i<j}\left(x_{i}-x_{j}\right), \\
& \Gamma(x)=\Gamma\left(x_{1}, \ldots, x_{k}\right)=\prod_{i<j}\left(x_{i}+x_{j}\right) .
\end{aligned}
$$

(When $k=0$ or 1 , we let $\Delta(x)=\Gamma(x)=1$. Likewise, $u_{0}=v_{0}=1$.) Then for $k \geq 1$ the following $k \times k$ determinant formulas hold:

$$
\begin{aligned}
& \mathcal{D}_{k}:=\left|\begin{array}{ccccc}
I_{10} & I_{11} & I_{12} & \cdots & I_{1, k-1} \\
I_{20} & I_{21} & I_{22} & \cdots & I_{2, k-1} \\
I_{30} & I_{31} & I_{32} & \cdots & I_{3, k-1} \\
\vdots & \vdots & \vdots & & \vdots \\
I_{k 0} & I_{k 1} & I_{k 2} & \cdots & I_{k, k-1}
\end{array}\right|=\frac{\left(u_{k}\right)^{2}}{2^{k}}, \\
& \mathcal{D}_{k}^{\prime}:=\left|\begin{array}{ccccc}
\beta_{0} & I_{10} & I_{11} & \cdots & I_{1, k-2} \\
\beta_{1} & I_{20} & I_{21} & \cdots & I_{2, k-2} \\
\beta_{2} & I_{30} & I_{31} & \cdots & I_{3, k-2} \\
\vdots & \vdots & \vdots & & \vdots \\
\beta_{k-1} & I_{k 0} & I_{k 1} & \cdots & I_{k, k-2}
\end{array}\right|=\frac{u_{k} u_{k-1}}{2^{k-1}}, \\
& \mathcal{D}_{k}^{\prime \prime}:=\left|\begin{array}{ccccc}
\beta_{11} & I_{12} & \cdots & I_{1, k-1} \\
\beta_{1} & I_{21} & I_{22} & \cdots & I_{2, k-1} \\
\beta_{2} & I_{31} & I_{32} & \cdots & I_{3, k-1} \\
\vdots & \vdots & \vdots & & \vdots \\
\beta_{k-1} & I_{k 1} & I_{k 2} & \cdots & I_{k, k-1}
\end{array}\right|=\frac{u_{k} v_{k-1}}{2^{k-1}} .
\end{aligned}
$$

The following lemmas state some additional determinant formulas needed in this paper. See Definition 4.8 for notation.

Lemma B.2. For $k \geq 1$,

$$
\mathcal{B}_{k}=\frac{1}{(2 k) !} \int_{\mathbf{R}^{2 k}} \frac{1}{\Gamma(x)}\left(\sum_{I} \Delta_{I}^{2} \Delta_{I^{c}}^{2} \Gamma_{I} \Gamma_{I^{c}}\right) \mathrm{d} \mu^{2 k}(x),
$$

where

$$
\Delta_{I}^{2}=\Delta\left(x_{i_{1}}, \ldots, x_{i_{k}}\right)^{2}, \quad \Gamma_{I}=\Gamma\left(x_{i_{1}}, \ldots, x_{i_{k}}\right),
$$

where the sum runs over all $\left(\begin{array}{c}2 k \\ k\end{array}\right)$ k-element subsets $I=\left\{i_{1}, \ldots, i_{k}\right\}$ of $\{1, \ldots, 2 k\}$, and where $I^{c}$ denotes the complement of I. Moreover, letting

$$
t_{k}=\frac{1}{k !} \int_{\mathbf{R}^{k}} \frac{\Delta(x)^{2}}{\Gamma(x)} \frac{\mathrm{d} \mu^{k}(x)}{x_{1} x_{2} \ldots x_{k}} \quad \text { for } k \geq 1
$$

we have

$$
\mathcal{B}_{k}=\frac{1}{2^{k}}\left|\begin{array}{cc}
t_{k} & u_{k-1} \\
t_{k+1} & u_{k}
\end{array}\right| .
$$

The proof of (B.7) is omitted since it is very similar to the proofs in [22]. Equation (B.10) follows by rewriting the integrand in (B.7) using the symmetric function identity (2.76) from [22. By simply replacing the measure $\mathrm{d} \mu(x)$ by $x \mathrm{~d} \mu(x)$ we obtain the corresponding formulas for $\mathcal{C}_{k}$ : 
Lemma B.3. For $k \geq 1$,

$$
\mathcal{C}_{k}=\frac{1}{(2 k) !} \int_{\mathbf{R}^{2 k}} \frac{x_{1} x_{2} \ldots x_{2 k}}{\Gamma(x)}\left(\sum_{I} \Delta_{I}^{2} \Delta_{I^{c}}^{2} \Gamma_{I} \Gamma_{I^{c}}\right) \mathrm{d} \mu^{2 k}(x) .
$$

Moreover,

$$
\mathcal{C}_{k}=\frac{1}{2^{k}}\left|\begin{array}{cc}
u_{k} & v_{k-1} \\
u_{k+1} & v_{k}
\end{array}\right| .
$$

It follows from (B.7) and (B.11) that if $\mathrm{d} \mu$ is a discrete measure supported on $n-1$ positive points, like in the main text, then $\mathcal{B}_{k}$ and $\mathcal{C}_{k}$ are strictly positive for $1 \leq k<n$ and zero for $k \geq n$ (because then each $\Delta_{I}$ vanishes).

Finally we state a formula for the determinant appearing in the proof of Corollary 4.18. We omit this proof as well; it is also similar to the previous ones (and actually a little easier, since no double integrals are involved).

Lemma B.4. Let the measure $\mathrm{d} \mu$ be the same as in the main text and define the $(n-1) \times(n-1)$ determinant $\mathcal{E}=\operatorname{det}\left[\mathcal{E}_{i j}\right]$ where

$$
\mathcal{E}_{i j}=\int \frac{x^{i}}{x+\lambda_{j}} \mathrm{~d} \mu(x), \quad i, j=1, \ldots, n-1 .
$$

Then

$$
\mathcal{E}=\frac{\Delta\left(\lambda_{n-1}, \lambda_{n-2}, \ldots, \lambda_{1}\right)}{(n-1) !} \int_{\mathbf{R}^{n-1}} \frac{\Delta(x)^{2}}{\prod_{i, j=1}^{n-1}\left(x_{i}+\lambda_{j}\right)} \mathrm{d} \mu^{n-1}(x) .
$$

In particular, $\mathcal{E} \neq 0$.

\section{References}

[1] Degasperis A and Procesi M 1999 in A Degasperis and G Gaeta, eds, Symmetry and perturbation theory (Rome, 1998) (River Edge, NJ: World Scientific Publishing) pp 23-37

[2] Degasperis A, Holm D D and Hone A N W 2002 A new integrable equation with peakon solutions Theoretical and Mathematical Physics 133 1463-1474 (Preprint nlin.SI/0205023)

[3] Johnson R S 2003 The classical problem of water waves: a reservoir of integrable and nearlyintegrable equations J. Nonlinear Math. Phys. 10(suppl. 1) 72-92

[4] Camassa R and Holm D D 1993 An integrable shallow water equation with peaked solitons Phys. Rev. Lett. 71(11) 1661-1664

[5] Hunter J K and Saxton R 1991 Dynamics of director fields SIAM J. Appl. Math. 51(6) 1498-1521

[6] Hunter J K and Zheng Y X 1994 On a completely integrable nonlinear hyperbolic variational equation Phys. D 79(2-4) 361-386

[7] Qiao Z and Li S 2004 A new integrable hierarchy, parametric solutions and traveling wave solutions Math. Phys. Anal. Geom. 7(4) 289-308

[8] Dullin H R, Gottwald G A and Holm D D 2003 Camassa-Holm, Korteweg-de Vries-5 and other asymptotically equivalent equations for shallow water waves Fluid Dynam. Res. 33(1-2) 73-95

[9] Rosenau P 1994 Nonlinear dispersion and compact structures Phys. Rev. Lett. 73(13) 1737-1741

[10] Fringer O B and Holm D D 2001 Integrable vs. nonintegrable geodesic soliton behavior Phys. D 150(3-4) 237-263

[11] Holm D D and Hone A N W 2005 A class of equations with peakon and pulson solutions $J$. Nonlinear Math. Phys. 12(suppl. 1) 380-394 With an appendix by H. W. Braden and J. G. Byatt-Smith

[12] Beals R, Sattinger D H and Szmigielski J 1998 Acoustic scattering and the extended Korteweg-de Vries hierarchy Adv. Math. 140(2) 190-206

[13] Krĕn M G 1952 On inverse problems for a nonhomogeneous cord Doklady Akad. Nauk SSSR (N.S.) $82669-672$

[14] Kreın M G 1952 On a generalization of investigations of Stieltjes Doklady Akad. Nauk SSSR (N.S.) $87881-884$ 
[15] Kac I S and Krein M G 1974 On the spectral functions of the string Amer. Math. Soc. Transl. 103(2) 19-102

[16] Stieltjes T J 1993 Euvres complètes/Collected papers. Vol. I, II (Berlin: Springer-Verlag)

[17] Beals R, Sattinger D H and Szmigielski J 1999 Multi-peakons and a theorem of Stieltjes Inverse Problems 15(1) L1-L4

[18] Beals R, Sattinger D H and Szmigielski J 2000 Multipeakons and the classical moment problem Adv. Math. 154(2) 229-257

[19] Beals R, Sattinger D H and Szmigielski J 2001 Inverse scattering solutions of the Hunter-Saxton equation Appl. Anal. 78(3-4) 255-269

[20] Dym H and McKean H P 1976 Gaussian processes, function theory, and the inverse spectral problem (New York: Academic Press [Harcourt Brace Jovanovich Publishers])

[21] Lundmark H and Szmigielski J 2003 Multi-peakon solutions of the Degasperis-Procesi equation Inverse Problems 19 1241-1245

[22] Lundmark H and Szmigielski J 2005 Degasperis-Procesi peakons and the discrete cubic string IMRP Int. Math. Res. Pap. 2005(2) 53-116

[23] Hone A N W and Wang J P 2003 Prolongation algebras and Hamiltonian operators for peakon equations Inverse Problems 19(1) 129-145

[24] Vakhnenko V A 1992 Solitons in a nonlinear model medium J. Phys. A 25(15) 4181-4187

[25] Vakhnenko V O and Parkes J 2004 in Symmetry in nonlinear mathematical physics. Part 1, 2, 3 vol 50 of Pr. Inst. Mat. Nats. Akad. Nauk Ukr. Mat. Zastos., Part 1 (Kiev: Natsīonal. Akad. Nauk Ukraïni Īnst. Mat.) pp 493-497

[26] Gantmacher F P and Krein M G 2002 Oscillation matrices and kernels and small vibrations of mechanical systems revised ed (AMS Chelsea Publishing, Providence, RI) Translation based on the 1941 Russian original, edited and with a preface by Alex Eremenko

[27] Fomin S and Zelevinsky A 2000 Total positivity: tests and parametrizations Math. Intelligencer 22(1) 23-33 